\title{
Computation of Fourier transform representations involving the generalized Bessel matrix polynomials
}

\author{
M. Abdalla $a^{1,2^{*}}$ and M. Ake $\mathrm{I}^{2,3}$
}

"Correspondence:

moabdalla@kku.edu.sa

${ }^{1}$ Department of Mathematics,

College of Science, King Khalid

University, P.O. Box 9004, 61413,

Abha, Saudi Arabia

2 Department of Mathematics,

Faculty of Science, South Valley

University, 83523 Qena, Egypt

Full list of author information is

available at the end of the article

\section{Springer}

\begin{abstract}
Motivated by the recent studies and developments of the integral transforms with various special matrix functions, including the matrix orthogonal polynomials as kernels, in this article we derive the formulas for Fourier cosine and sine transforms of matrix functions involving generalized Bessel matrix polynomials. With the help of these transforms several results are obtained, which are extensions of the corresponding results in the standard cases. The results given here are of general character and can yield a number of (known and new) results in modern integral transforms.
\end{abstract}

MSC: 42A38; 44A05; 44A20; 35S30

Keywords: Fourier cosine transforms; Fourier sine transforms; Generalized Bessel matrix polynomials; Operational calculus

\section{Introduction}

In the past few decades, the orthogonal matrix polynomials have attracted a lot of research interest due to their close relations and various applications in many areas of mathematics, engineering, probability theory, graph theory, and physics; for example, see [1-9]. In [4], extensions to the matrix framework of the classical families of Legendre, Laguerre, Jacobi, Chebyshev, Gegenbauer, and Hermite polynomials have been introduced. Meanwhile, one particular orthogonal polynomial family which frequently appears in the recent studies and applications [10-12] is that of generalized Bessel polynomials, which in its matrix form is also defined in $[4,13]$. Later on, distinct works on the generalized Bessel matrix polynomials have been discussed (see [14-17]).

Nowadays, many integral transforms (see, e.g., Fourier, Laplace, Beta, Hankel, Mellin, Whittaker transforms, etc.), with various special functions (also with the new generalized special matrix functions) as kernels, have begun to play an important role in modeling of various physical, engineering, automatization, and biological phenomena, as well as in several other branches of science (see, for instance, [8, 18-30]).

Fourier transform (FT) is an integral transform that is used in solving different problems in mathematical physics, applied statistics, and engineering (see, [31, 32]). The idea

(c) The Author(s) 2021. This article is licensed under a Creative Commons Attribution 4.0 International License, which permits use sharing, adaptation, distribution and reproduction in any medium or format, as long as you give appropriate credit to the original author(s) and the source, provide a link to the Creative Commons licence, and indicate if changes were made. The images or other third party material in this article are included in the article's Creative Commons licence, unless indicated otherwise in a credit line to the material. If material is not included in the article's Creative Commons licence and your intended use is not permitted by statutory regulation or exceeds the permitted use, you will need to obtain permission directly from the copyright holder. To view a copy of this licence, visit http://creativecommons.org/licenses/by/4.0/. 
of Fourier transform is a natural extension of the idea of Fourier series. In particular, Fourier transform can accommodate aperiodic functions, which Fourier series cannot do. Recently, many results on Fourier transform and its applications have been contributed by Nicola and Trapasso [33], Urieles et al. [34], Ghodadra and Fülöp [35], Bergold and Lasser [36], and Al-Lail and Qadir [37].

On the contrary, matrix Fourier expansions and Fourier series in orthonormal matrix polynomials have been introduced by B. Osihnker in [38, 39]. Defez and Jóbdar [40, 41] introduced basic properties of matrix Fourier series and Fourier approximation for functions of matrix argument. Recently, Groenevelt and Koelink [42] discussed the generalized Fourier transform with hypergeometric function and matrix-valued orthogonal polynomials as kernels. Also, applications of matrix summability to Fourier transforms were established by Ş. Yildiz [43].

Motivated by some of these aforementioned investigations of the Fourier transforms of matrix-valued orthogonal polynomials, in our investigation here we study the Fouriertype transforms of the generalized Bessel matrix polynomials $\mathcal{Y}_{n}(\xi ; F, L), \xi \in \mathbb{C}$, for (square) matrix parameters $F$ and $L$. In particular, we obtain several Fourier cosine and sine transforms of functions involving generalized Bessel matrix polynomials with powers of the matrix, as well as matrix exponential, trigonometric, binomial, and Bessel functions. Moreover, pertinent integral transforms of the different results given here, including simpler and earlier ones, are also investigated.

\section{Auxiliary toolbox}

In this section, we recall some definitions, lemmas, and terminology which will be used to prove the main results.

Let $\mathbb{C}$ and $\mathbb{N}$ denote the sets of complex numbers and positive integers, respectively, and $\mathbb{N}_{0}=\mathbb{N} \cup\{0\}$. Let $\mathbb{C}^{n}$ denote the $n$-dimensional complex vector space and $\mathbb{C}^{n \times n}$ denote the space of all square matrices with $n$ rows and $n$ columns whose entries are complex numbers.

Definition 2.1 ([4]) For a matrix $F$ in $\mathbb{C}^{n \times n}$, the spectrum $\sigma(F)$ is the set of all eigenvalues of $F$ for which we denote

$$
\alpha(F)=\max \{\operatorname{Re}(\xi): \xi \in \sigma(F)\} \quad \text { and } \quad \widetilde{\alpha}(F)=\min \{\operatorname{Re}(\xi): \xi \in \sigma(F)\}
$$

where $\alpha(F)$ refers to the spectral abscissa of $F$ and for which $\widetilde{\alpha}(F)=-\alpha(-F)$. A matrix $F$ is said to be positive stable if and only if $\widetilde{\alpha}(F)>0$.

Definition 2.2 ([44]) If $F$ and $L$ are commuting matrices in $\mathbb{C}^{n \times n}$ and $w \in \mathbb{C}$, then

$$
\begin{aligned}
& \cos [(F \pm L) w]=\cos (F w) \cos (L w) \mp \sin (F w) \sin (L w) \\
& \sin [(F \pm L) w]=\sin (F w) \cos (L w) \pm \cos (F w) \sin (L w)
\end{aligned}
$$

Remark 2.1 If $F, L \in \mathbb{C}^{1 \times 1}=\mathbb{C}$, then the identities in Definition 2.2 reduce to those in the scalar setting. 
Definition $2.3([4,45])$ Let $F$ be a positive stable matrix in $\mathbb{C}^{n \times n}$. The gamma matrix function $\Gamma(F)$ is defined as

$$
\Gamma(F)=\int_{0}^{\infty} e^{-w} w^{F-I} d w ; \quad w^{F-I}=\exp ((F-I) \ln w)
$$

where $I$ is the identity matrix in $\mathbb{C}^{n \times n}$.

Definition $2.4([4,45])$ The reciprocal gamma function denoted by $\Gamma^{-1}(w)=\frac{1}{\Gamma(w)}$ is an entire function of the complex variable $\xi$. Then the image of $\Gamma^{-1}(w)$ acting on $F \in \mathbb{C}^{n \times n}$ denoted by $\Gamma^{-1}(F)$ is a well-defined matrix and invertible, as well as

$$
F+n I \text { is invertible for all integers } n \in \mathbb{N}_{0} \text {. }
$$

By applying the matrix functional calculus to $F$, which is a positive stable matrix in $\mathbb{C}^{n \times n}$, the Pochhammer symbol of a matrix argument defined by

$$
(F)_{n}= \begin{cases}F(F+I) \cdots(F+(n-1) I)=\Gamma^{-1}(F) \Gamma(F+n I), & n \geq 1 \\ I, & n=0 .\end{cases}
$$

Note that, if $F=-s I$, where $s$ is a positive integer, then $(F)_{n}=0$, whenever $n>s$.

Now, from properties of the gamma matrix function, we give some lemmas which will be needed in the proof of some theorems.

Lemma 2.1 Let $S$ be a matrix in $\mathbb{C}^{n \times n}$ such that $\widetilde{\alpha}(S)>0$ and $w \in \mathbb{C}$ with $\operatorname{Re}(w)>0$. The following integral formulas hold:

$$
\int_{0}^{\infty} \xi^{S-I} e^{-w \xi} d \xi=w^{-S} \Gamma(S)
$$

and

$$
\int_{0}^{\infty} \xi^{S-I} e^{-w i \xi} d \xi=e^{-\frac{1}{2} i \pi S} w^{-S} \Gamma(S) ; \quad i=\sqrt{-1}
$$

We thus observe that

$$
\int_{0}^{\infty} \xi^{S-I} \cos (w \xi) d \xi=\cos \left(\frac{1}{2} \pi S\right) w^{-S} \Gamma(S)
$$

and

$$
\int_{0}^{\infty} \xi^{S-I} \sin (w \xi) d \xi=\sin \left(\frac{1}{2} \pi S\right) w^{-S} \Gamma(S) .
$$

Putting $S=I-R \in \mathbb{C}^{n \times n}$ in (8) and (9), we get

$$
\int_{0}^{\infty} \xi^{-R} \cos (w \xi) d \xi=\frac{\pi w^{R-I}}{2} \sec \left(\frac{1}{2} \pi R\right) \Gamma^{-1}(R), \quad \widetilde{\alpha}(R)>0,
$$


and

$$
\int_{0}^{\infty} \xi^{-R} \sin (w \xi) d \xi=\frac{\pi w^{R-I}}{2} \csc \left(\frac{1}{2} \pi R\right) \Gamma^{-1}(R), \quad \widetilde{\alpha}(R)>0 .
$$

Similarly, we can present the following lemma.

Lemma 2.2 Let $S$ be a matrix in $\mathbb{C}^{n \times n}$ such that $\widetilde{\alpha}(S)>0, \lambda, w \in \mathbb{C}$ with $\operatorname{Re}(\lambda)>0$ and $\operatorname{Re}(w)>0$. The following integral formulas hold:

$$
\int_{0}^{\infty} \xi^{S-I} e^{-\lambda \xi} \cos (w \xi) d \xi=\cos \left(\arctan \left(\frac{w}{\lambda}\right) S\right)\left(\lambda^{2}+w^{2}\right)^{-\frac{1}{2} S} \Gamma(S)
$$

and

$$
\int_{0}^{\infty} \xi^{S-I} e^{-\lambda \xi} \sin (w \xi) d \xi=\sin \left(\arctan \left(\frac{w}{\lambda}\right) S\right)\left(\lambda^{2}+w^{2}\right)^{-\frac{1}{2} S} \Gamma(S)
$$

Definition $2.5([4,46])$ Let $k$ and $r$ be finite positive integers. The generalized hypergeometric matrix function is defined by the matrix power series

$$
{ }_{k} \mathbf{H}_{r}[\mathbf{F} ; \mathbf{L} ; w]=\sum_{m=0}^{\infty} \prod_{i=1}^{k}\left(F_{i}\right)_{m} \prod_{j=1}^{r}\left[\left(L_{j}\right)_{m}\right]^{-1} \frac{w^{m}}{m !}
$$

where $\mathbf{F}=F_{i}, 1 \leq i \leq k$, and $\mathbf{L}=L_{j}, 1 \leq j \leq r$, are commutative matrices in $\mathbb{C}^{n \times n}$ with $L_{j}+m I$ being invertible for all integers $m \in \mathbb{N}_{0}$.

Note that for $k=1, r=0$, we have the binomial-type matrix function ${ }_{1} \mathbf{H}_{0}\left(F_{1} ;-; w\right),|w|<$ 1, as follows:

$$
{ }_{1} \mathbf{H}_{0}(F ;-; w)=(1-w)^{-F_{1}}=I+F_{1} w+\frac{F_{1}\left(F_{1}+I\right) w^{2}}{2 !}+\cdots+\frac{\left(F_{1}\right)_{n} w^{n}}{n !}+\cdots
$$

Also, note that for $k=2, r=1$, we get the Gauss hypergeometric matrix function ${ }_{2} \mathbf{H}_{1}$ in the form

$$
{ }_{2} \mathbf{H}_{1}\left(F_{1}, F_{2} ; L_{1} ; w\right)=\sum_{s=0}^{\infty}\left(F_{1}\right)_{s}\left(F_{2}\right)_{s}\left[\left(L_{1}\right)_{s}\right]^{-1} \frac{w^{s}}{s !} .
$$

Several special matrix functions, including the matrix orthogonal polynomials, are also presented in terms of the generalized hypergeometric matrix function in $[4,46]$.

Definition $2.6([4,13,16])$ Let $F$ and $L$ be commuting matrices in $\mathbb{C}^{n \times n}$ such that $L$ is an invertible matrix. For any natural number $n \in \mathbb{N}_{0}$ and $\xi \in \mathbb{C}$, the $n$th generalized Bessel 
matrix polynomial $\mathcal{Y}_{n}(\xi ; F, L)$ is defined as

$$
\begin{aligned}
\mathcal{Y}_{n}(\xi ; F, L) & =\sum_{s=0}^{n} \frac{(-1)^{s}}{s !}(-n I)_{s}(F+(n-1) I)_{s}\left(\xi L^{-1}\right)^{s} \\
& =\sum_{s=0}^{n} \frac{n}{s !(n-s) !}(F+(n-1) I)_{s}\left(\xi L^{-1}\right)^{s} \\
& ={ }_{2} \mathbf{H}_{0}\left[\begin{array}{c}
-n I, F+(n-1) I \\
-
\end{array}-\xi L^{-1}\right] .
\end{aligned}
$$

Remark 2.2 If the matrices $F, L \in \mathbb{C}^{1 \times 1}=\mathbb{C}$, then the generalized Bessel matrix polynomial in (15) reduces to generalized Bessel polynomials in [10-12].

Definition 2.7 $([47,48])$ Let a matrix $F \in \mathbb{C}^{n \times n}$ satisfy the condition:

$$
\beta \text { is not a negative integer for every } \beta \in \sigma(F) \text {, }
$$

then Bessel matrix function $\mathbf{J}_{F}(w)$ of the first kind associated to $F$ is given by

$$
\mathbf{J}_{F}(w)=\sum_{s=0}^{\infty} \frac{(-1)^{s}}{(s) !} \Gamma^{-1}(F+(s+1) I)\left(\frac{w}{2}\right)^{F+2 s I}, \quad w \in \mathbb{C},
$$

and the modified Bessel matrix functions $\mathbf{I}_{F}(w)$ and $\mathbf{K}_{F}(w)$ are respectively defined as

$$
\mathbf{I}_{F}(w)=\sum_{s=0}^{\infty} \frac{1}{(s) !} \Gamma^{-1}(F+(s+1) I)\left(\frac{w}{2}\right)^{F+2 s I}
$$

and

$$
\mathbf{K}_{F}(w)=\frac{\pi}{2}[\sin (\pi F)]^{-1}\left\{\mathbf{I}_{-F}(w)-\mathbf{I}_{F}(w)\right\} .
$$

Definition $2.8([31,32])$ Let $f(\xi)$ be a function of $\xi$ specified for $\xi>0$. Then the complex Fourier transform of $f(\xi)$ associated with complex frequency $w$ is defined by

$$
\mathcal{F}(w)=\mathcal{F}\{f(\xi)\}=\frac{1}{\sqrt{2 \pi}} \int_{-\infty}^{\infty} f(\xi) e^{-i \xi w} d \xi, \quad w \in \mathbb{C},
$$

together with the requirement of $|\mathcal{F}(w)|<\infty$.

Similarly, the inverse Fourier transform, denoted by $\mathcal{F}^{-1}\{\mathcal{F}(w)\}=f(\xi)$, is defined by

$$
f(\xi)=\mathcal{F}^{-1}\{\mathcal{F}(w)\}=\frac{1}{\sqrt{2 \pi}} \int_{-\infty}^{\infty} \mathcal{F}(w) e^{i \xi w} d w
$$

The cosine and sine transformations, respectively, are defined similarly as follows:

$$
\mathcal{F}^{c}(w)=\sqrt{\frac{2}{\pi}} \int_{0}^{\infty} f(\xi) \cos (\xi w) d \xi
$$




$$
\begin{aligned}
& f(\xi)=\sqrt{\frac{2}{\pi}} \int_{0}^{\infty} \mathcal{F}^{c}(w) \cos (\xi w) d w, \\
& \mathcal{F}^{s}(w)=\sqrt{\frac{2}{\pi}} \int_{0}^{\infty} f(\xi) \sin (\xi w) d \xi
\end{aligned}
$$

and

$$
f(\xi)=\sqrt{\frac{2}{\pi}} \int_{0}^{\infty} \mathcal{F}^{s}(w) \sin (\xi w) d w .
$$

Note that if $f(\xi)$ is an even function, then $\mathcal{F}(w)=\mathcal{F}^{c}(w)$, and if $f(\xi)$ is an odd function, then $\mathcal{F}(w)=i \mathcal{F}^{s}(w)$.

The following lemma will be required in the proof of our theorems.

Lemma 2.3 ([18]) From the basic formulae of the Fourier cosine transform, if $f(\xi)$ is replaced by $\xi^{2 n} f(\xi)$, then

$$
\mathcal{F}^{c}\left\{\xi^{2 n} f(\xi)\right\}(w)=(-1)^{n} \frac{d^{2 n}}{d w^{2 n}}\left(\mathcal{F}^{c}\{f\}(w)\right)
$$

Also, if

$$
f(\xi)=\left(\lambda^{2}+\xi^{2}\right)^{-\left(S+\frac{1}{2}\right)} ; \quad \widetilde{\alpha}(S)>-\frac{1}{2},
$$

then

$$
\mathcal{F}^{c}(w)=\sqrt{2}(w / 2 \lambda)^{S} \Gamma^{-1}\left(S+\frac{1}{2} I\right) K_{S}(\lambda w)
$$

where $S$ is a positive stable matrix in $\mathbb{C}^{n \times n}, w, \lambda \in \mathbb{C}$ with $\operatorname{Re}(w)>0, \operatorname{Re}(\lambda)>0$, and $\boldsymbol{K}_{S}(w)$ is the modified Bessel matrix function in (19).

Remark 2.3 Physically, the Fourier transform $\mathcal{F}(w)$ can be interpreted as an integral superposition of an infinite number of sinusoidal oscillations with different wavenumbers $w$ (or different wavelengths $\tau=\frac{2 \pi}{w}$ ). Thus, the definition of the Fourier transform is restricted to absolutely integrable functions. This restriction is too strong for many physical applications (see [31, 32]).

\section{Statement and proof of main theorems}

In this section, we investigate several new interesting Fourier cosine and sine transforms of functions involving generalized Bessel matrix polynomials asserted in the following theorems:

Theorem 3.1 Let $S, F$ and $L$ be commuting matrices in $\mathbb{C}^{n \times n}$, and let $\mathcal{Y}_{n}(\lambda \xi ; F, L)$ be given in (15). For the function

$$
f(\xi)=\xi^{S} \mathcal{Y}_{n}(\lambda \xi ; F, L)
$$


we have

$$
\begin{aligned}
\mathcal{F}^{c}(w)= & -\sqrt{\frac{2}{\pi}} w^{-(S+I)} \Gamma(S+I) \sum_{r=0}^{n}(-n I)_{r}\left(F+(n-1)_{I}\right)_{r}(S+I)_{r} \\
& \times \frac{\left(-\lambda(L w)^{-1}\right)^{r} \sin [(S+r I) \pi / 2]}{r !}, \\
\mathcal{F}^{s}(w)= & \sqrt{\frac{2}{\pi}} w^{-(S+I)} \Gamma(S+I) \sum_{r=0}^{n}(-n I)_{r}(F+(n-1) I)_{r}(S+I)_{r} \\
& \times \frac{\left(-\lambda(L w)^{-1}\right)^{r} \cos [(S+r I) \pi / 2]}{r !}
\end{aligned}
$$

where $w, \lambda \in \mathbb{C}$ are such that $\operatorname{Re}(w)>0, \operatorname{Re}(\lambda)>0$, and $\widetilde{\alpha}(S)>-1$.

Proof To prove (25) from Definition 2.6 and (22), we observe that

$$
\begin{aligned}
\mathcal{F}^{c}(w) & =\sqrt{\frac{2}{\pi}} \int_{0}^{\infty} \xi^{S} \mathcal{Y}_{n}(\lambda \xi ; F, L) \cos (\xi w) d \xi \\
& =\sqrt{\frac{2}{\pi}} \sum_{r=0}^{n}(-n I)_{r}(F+(n-1) I)_{r} \frac{\left(-\lambda L^{-1}\right)^{r}}{r !} \int_{0}^{\infty} \xi^{S+r I} \cos (w \xi) d \xi .
\end{aligned}
$$

From the integral (8), we obtain

$$
\begin{aligned}
\mathcal{F}^{c}(w)= & \sqrt{\frac{2}{\pi}} \sum_{r=0}^{n}(-n I)_{r}(F+(n-1) I)_{r} \frac{\left(-\lambda L^{-1}\right)^{r}}{r !} \\
& \times w^{-(S+(r+1) I)} \Gamma(S+(r+1) I) \cos [(S+(r+1) I) \pi / 2] \\
= & -\sqrt{\frac{2}{\pi}} w^{-(S+I)} \Gamma(S+I) \sum_{r=0}^{n}(-n I)_{r}(F+(n-1) I)_{r} \\
& \times(S+I)_{r} \frac{\left(-\lambda(w L)^{-1}\right)^{r}}{r !} \sin [(S+r I) \pi / 2],
\end{aligned}
$$

which is the claimed result in (25).

Now, we prove (26), from (24) in (23), we have

$$
\begin{aligned}
\mathcal{F}^{s}(w) & =\sqrt{\frac{2}{\pi}} \int_{0}^{\infty} \xi^{S} \mathcal{Y}_{n}(\lambda \xi ; F, L) \sin (\xi w) d \xi \\
& =\sqrt{\frac{2}{\pi}} \sum_{r=0}^{n}(-n I)_{r}(F+(n-1) I)_{r} \frac{\left(-\lambda L^{-1}\right)^{r}}{r !} \int_{0}^{\infty} \xi^{S+r I} \sin (w \xi) d \xi
\end{aligned}
$$

By invoking relation (9), we obtain

$$
\begin{aligned}
\mathcal{F}^{s}(w)= & \sqrt{\frac{2}{\pi}} \sum_{r=0}^{n}(-n I)_{r}(F+(n-1) I)_{r} \frac{\left(-\lambda L^{-1}\right)^{r}}{r !} \\
& \times w^{-(S+(r+1) I)} \Gamma(S+(r+1) I) \sin [(S+(r+1) I) \pi / 2]
\end{aligned}
$$




$$
\begin{aligned}
= & -\sqrt{\frac{2}{\pi}} w^{-(S+I)} \Gamma(S+I) \sum_{r=0}^{n}(-n I)_{r}(F+(n-1) I)_{r} \\
& \times(S+I)_{r} \frac{\left(-\lambda(w L)^{-1}\right)^{r}}{r !} \cos [(S+r I) \pi / 2],
\end{aligned}
$$

which is the desired result in (26).

Now by taking advantage of the previous results, we obtain the following corollaries:

Corollary 3.1.1 If $r$ is odd, then (25) reduces to

$$
\begin{aligned}
\mathcal{F}^{c}(w)= & \sqrt{\frac{2}{\pi}} w^{-(S+I)} \Gamma(S+I) \cos (S \pi / 2) \\
& \times \sum_{r=1}^{n}(-n I)_{r}(F+(n-1) I)_{r}(S+I)_{r} \frac{(-1)^{\frac{r+1}{2}}\left(-\lambda(w L)^{-1}\right)^{r}}{r !}
\end{aligned}
$$

and if $r$ is even, then (25) reduces to

$$
\begin{aligned}
\mathcal{F}^{c}(w)= & -\sqrt{\frac{2}{\pi}} w^{-(S+I)} \Gamma(S+I) \sin (S \pi / 2) \\
& \times \sum_{r=0}^{n}(-n I)_{r}(F+(n-1) I)_{r}(S+I)_{r} \frac{(-1)^{\frac{r}{2}}\left(-\lambda(w L)^{-1}\right)^{r}}{r !} .
\end{aligned}
$$

Corollary 3.1.2 If $r$ is odd, then (26) reduces to

$$
\begin{aligned}
\mathcal{F}^{s}(w)= & \sqrt{\frac{2}{\pi}} w^{-(S+I)} \Gamma(S+I) \sin (S \pi / 2) \\
& \times \sum_{r=1}^{n}(-n I)_{r}(F+(n-1) I)_{r}(S+I)_{r} \frac{(-1)^{\frac{r+1}{2}}\left(-\lambda(w L)^{-1}\right)^{r}}{r !}
\end{aligned}
$$

and if $r$ is even, then (26) reduces to

$$
\begin{aligned}
\mathcal{F}^{s}(w)= & \sqrt{\frac{2}{\pi}} w^{-(S+I)} \Gamma(S+I) \cos (S \pi / 2) \\
& \times \sum_{r=0}^{n}(-n I)_{r}(F+(n-1) I)_{r}(S+I)_{r} \frac{(-1)^{\frac{r}{2}}\left(-\lambda(w L)^{-1}\right)^{r}}{r !} .
\end{aligned}
$$

Corollary 3.1.3 Replacing the Bessel matrix polynomials $\mathcal{Y}_{n}(\lambda \xi ; F, L)$ by $\mathcal{Y}_{n}\left(\lambda \xi^{2} ; F, L\right)$ and choosing $S=0$ in (25) and (26), that is, if

$$
f(\xi)=\mathcal{Y}_{n}\left(\lambda \xi^{2} ; F, L\right)
$$

then, we obtain the following results:

$$
\mathcal{F}^{c}(w)=0,
$$


and

$$
\mathcal{F}^{S}(w)=\sqrt{\frac{2}{\pi}} w_{4}^{-1} \mathcal{H}_{0}\left[\begin{array}{c}
-n I, F+(n-1) I, \frac{1}{2} I, I \\
-
\end{array} ; 4 \lambda\left(L w^{2}\right)^{-1}\right] .
$$

Also, a consequence of Theorem 3.1 is the following theorem:

Theorem 3.2 Let $S, F$, and $L$ be commuting matrices in $\mathbb{C}^{n \times n}$, and let $\mathcal{Y}_{n}(\lambda \xi ; F, L)$ be given in (15). For the function

$$
f(\xi)=\xi^{S} \cos (\mu \xi) \mathcal{Y}_{n}(\lambda \xi ; F, L),
$$

we have

$$
\begin{aligned}
\mathcal{F}^{c}(w)= & \frac{-1}{2} \sqrt{\frac{2}{\pi}} \Gamma(I+S) \\
& \times \sum_{r=0}^{n}(-n I)_{r}(F+(n-1) I)(S+I)_{r} \frac{\left(\lambda L^{-1}\right)^{r} \sin [(S+r I) \pi / 2]}{r !} \\
& \times\left\{(w+\mu)^{-(S+(r+1) I)}+|(w-\mu)|^{-(S+(r+1) I)}\right\},
\end{aligned}
$$

and

$$
\begin{aligned}
\mathcal{F}^{S}(w)= & \frac{1}{2} \sqrt{\frac{2}{\pi}} \Gamma(I+S) \\
& \times \sum_{r=0}^{n}(-n I)_{r}(F+(n-1) I)(S+I)_{r} \frac{\left(\lambda L^{-1}\right)^{r} \cos [(S+r I) \pi / 2]}{r !} \\
& \times\left\{(w+\mu)^{-(S+(r+1) I)}+|w-\mu|^{-(S+(r+1) I)}\right\}
\end{aligned}
$$

where $w, \mu, \lambda \in \mathbb{C}$ are such that $\operatorname{Re}(w)>0, \operatorname{Re}(\mu)>0, \operatorname{Re}(\lambda)>0, \operatorname{Re}(w)>\operatorname{Re}(\mu)$, and $\tilde{\alpha}(S+$ I) $>0$.

Proof To describe the relation in (28), the proof is easy, using the well-known identities in (2). In a similar way, we can get the result in (29).

Theorem 3.3 Let $S, F$, and $L$ be commuting matrices in $\mathbb{C}^{n \times n}$. If

$$
f(\xi)=\xi^{-S} \mathcal{Y}_{n}(\lambda ; F, L \xi)
$$

then,

$$
\begin{aligned}
\mathcal{F}^{c}(w)= & \sqrt{\frac{2}{\pi}} w^{(S-I)} \Gamma(I-S) \\
& \times \sum_{r=0}^{n}(-n I)_{r}(F+(n-1) I)\left[(S)_{r}\right]^{-1} \frac{\left(\lambda w L^{-1}\right)^{r} \sin [(S+r I) \pi / 2]}{r !},
\end{aligned}
$$


and

$$
\begin{aligned}
\mathcal{F}^{S}(w)= & \sqrt{\frac{2}{\pi}} w^{(S-I)} \Gamma(I-S) \\
& \times \sum_{r=0}^{n}(-n I)_{r}(F+(n-1) I)\left[(S)_{r}\right]^{-1} \frac{\left(\lambda w\left(L^{-1}\right)^{r} \cos [(S+r I) \pi / 2]\right.}{r !},
\end{aligned}
$$

where $\operatorname{Re}(w)>0, \operatorname{Re}(\lambda)>0$, and $\widetilde{\alpha}(I-S)>0$.

Proof The proofs of the two results in (31) and (32) can be obtained by the use of the two formulas in (10) and (11) with Definition 2.6.

Theorem 3.4 Let $S, F$, and $L$ be commuting matrices in $\mathbb{C}^{n \times n}$, and let $\mathcal{Y}_{n}(\lambda \xi ; F, L)$ be given in (15). For the function

$$
f(\xi)=\xi^{S-I} e^{-\mu \xi} \mathcal{Y}_{n}(\lambda \xi ; F, L)
$$

we have

$$
\begin{aligned}
\mathcal{F}^{c}(w)= & \sqrt{\frac{2}{\pi}}\left(\mu^{2}+w^{2}\right)^{\frac{-S}{2}} \Gamma(S) \\
& \times \sum_{r=0}^{n}(-n I)_{r}(F+(n-1) I)_{r}(S)_{r} \frac{\left(-\lambda L^{-1}\right)^{r} \cos [(S+r I) \arctan (w / \mu)]}{r !},
\end{aligned}
$$

and

$$
\begin{aligned}
\mathcal{F}^{S}(w)= & \sqrt{\frac{2}{\pi}}\left(\mu^{2}+w^{2}\right)^{\frac{-S}{2}} \Gamma(S) \\
& \times \sum_{r=0}^{n}(-n I)_{r}(F+(n-1) I)_{2}(S)_{r} \frac{\left(-\lambda L^{-1}\right)^{r} \sin [(S+r I) \arctan (w / \mu)]}{r !},
\end{aligned}
$$

where $w, \mu, \lambda \in \mathbb{C}$ are such that $\operatorname{Re}(w)>0, \operatorname{Re}(\mu)>0, \operatorname{Re}(\lambda)>0$, and $\widetilde{\alpha}(S)>0$.

Proof Using (15) and applying formula (22) on the right-hand side of (33) reveals that

$$
\begin{aligned}
\mathcal{F}^{c}(w)= & \sqrt{\frac{2}{\pi}} \int_{0}^{\infty} \xi^{S-I} e^{-\mu \xi} \mathcal{Y}_{n}(\lambda \xi ; F, L) \cos (\xi w) d \xi \\
= & \sqrt{\frac{2}{\pi}} \sum_{r=0}^{n}(-n I)_{r}(F+(n-1) I)_{r} \frac{\left(-\lambda L^{-1}\right)^{r}}{r !} \\
& \times \int_{0}^{\infty} \xi^{S-(1-r) I} e^{-\mu \xi} \cos (\xi w) d \xi
\end{aligned}
$$

Using (12), we get

$$
\begin{aligned}
\mathcal{F}^{c}(w)= & \sqrt{\frac{2}{\pi}} \sum_{r=0}^{n}(-n I)_{r}(F+(n-1) I)_{r} \frac{\left(-\lambda L^{-1}\right)^{r}}{r !} \\
& \times\left(\mu^{2}+w^{2}\right)^{\frac{-1}{2} S} \Gamma(S+r I) \cos [(S+r I) \arctan (w / \mu)],
\end{aligned}
$$

which implies formula (34). 
Likewise, we can get the result in (35) by using (13).

\section{Theorem 3.5 For the function}

$$
f(\xi)=\xi^{2 n} e^{-\mu \xi^{2}} \mathcal{Y}_{n}\left(\lambda \xi^{2} ; F, L\right)
$$

where $\mathcal{Y}_{n}(-; F, L)$ is as in (15), we have

$$
\begin{aligned}
\mathcal{F}^{c}(w)= & \sqrt{\frac{1}{2 \pi}} \Gamma(n+1 / 2) \mu^{-\left(\frac{1}{2}+2 n\right)}\left(-w^{2} / 4\right)^{n} e^{\left(-w^{2} / 4 \mu\right)} \\
& \times \sum_{r=0}^{n}(-n I)_{r}(F+(n-1) I)_{r} \frac{\left(\lambda w^{2}\left(4 \mu^{2} L\right)^{-1}\right)^{r}}{r !} \\
& \times \mathcal{Y}_{n+r}\left(4 \mu ; 3 / 2-2(n+r), w^{2}\right),
\end{aligned}
$$

where $w, \mu, \lambda \in \mathbb{C}$ are such that $\operatorname{Re}(w)>0, \operatorname{Re}(\mu)>0$, and $\operatorname{Re}(\lambda)>0$.

Proof To establish our result in (37), using (36) in (22), we arrive at

$$
\begin{aligned}
\mathcal{F}^{c}(w)= & \sqrt{\frac{2}{\pi}} \int_{0}^{\infty} \xi^{2 n} e^{-\mu \xi^{2}} \mathcal{Y}_{n}\left(\lambda \xi^{2} ; F, L\right) \cos (\xi w) d \xi \\
= & \sqrt{\frac{2}{\pi}} \sum_{r=0}^{n}(-n I)_{r}(F+(n-1) I)_{r} \frac{\left(-\lambda L^{-1}\right)^{r}}{r !} \\
& \times \int_{0}^{\infty} \xi^{2 n+2 r} e^{-\mu \xi^{2}} \sum_{s=0}^{\infty} \frac{\left(-w^{2} \xi^{2}\right)^{s}}{(2 s) !} d \xi \\
= & \sqrt{\frac{1}{2 \pi}} \sum_{r=0}^{n} \sum_{s=0}^{\infty}(-n I)_{r}(F+(n-1) I)_{r} \frac{\left(-w^{2}\right)^{s}\left(-\lambda L^{-1}\right)^{r}}{\Gamma(2 s+1) r !} \\
& \times \int_{0}^{\infty} \xi^{n+r+s-\frac{1}{2}} e^{-\mu \xi} d \xi \\
= & \sqrt{\frac{1}{2 \pi}} \sum_{r=0}^{n} \sum_{s=0}^{\infty}(-n I)_{r}(F+(n-1) I)_{r} \frac{\left(-\lambda L^{-1}\right)^{r}}{r !} \\
& \times \frac{\sqrt{\pi} \Gamma\left(n+r+s+\frac{1}{2}\right)}{\Gamma\left(\frac{1}{2}\right)\left(\frac{1}{2}\right)_{s} s ! 2^{2 s} \mu^{\left(n+r+s+\frac{1}{2}\right)}}\left(-w^{2}\right)^{s} .
\end{aligned}
$$

After changing the order of summation and simplifying, we obtain

$$
\begin{aligned}
\mathcal{F}^{c}(w)= & \sum_{s=0}^{\infty} \frac{\left(n+r+\frac{1}{2}\right)_{s}\left(-w^{2} / 4 \mu\right)^{s}}{s !\left(\frac{1}{2}\right)_{s}} \\
= & \sqrt{\frac{1}{2 \pi}} \mu^{-\left(n+\frac{1}{2}\right)} \Gamma\left(n+\frac{1}{2}\right) e^{\left(-w^{2} / 4 \mu\right)} \\
& \times \sum_{r=0}^{n}(-n I)_{r}(F+(n-1) I)_{r}\left(n+\frac{1}{2}\right)_{r} \frac{\left(-\lambda(\mu L)^{-1}\right)^{r}}{r !} \\
& \times \frac{\left(-w^{2} / 4 \mu\right)^{n+r}}{\left(\frac{1}{2}\right)_{n+r}} \sum_{s=0}^{n+r}(-n-r)_{s}\left(\frac{1}{2}-n-r\right)_{s} \frac{\left(-4 \mu / w^{2}\right)^{s}}{s !}
\end{aligned}
$$

which implies formula (37). 
Theorem 3.6 Let $\mathcal{Y}_{n}(\lambda \xi ; F, L)$ be given in (15). For the function

$$
f(\xi)=\xi^{2 n+1} e^{-\mu \xi^{2}} \mathcal{Y}_{n}\left(\lambda \xi^{2} ; F, L\right)
$$

we have

$$
\begin{aligned}
\mathcal{F}^{s}(w)= & \frac{1}{2 \sqrt{2}} \mu^{-(2 n+3 / 2)}\left(-w^{2} / 4\right)^{n} e^{\left(-w^{2} / 4 \mu\right)} \\
& \times \sum_{r=0}^{n}(-n I)_{r}(F+(n-1) I)_{r} \frac{\left(\lambda w\left(4 \mu^{2} L\right)^{-1}\right)^{r}}{r !} \mathcal{Y}_{n+r}\left(4 \mu ; \frac{1}{2}-2(n+r), w^{2}\right),
\end{aligned}
$$

where $w, \mu, \lambda \in \mathbb{C}$ are such that $\operatorname{Re}(w)>0, \operatorname{Re}(\mu)>0$, and $\operatorname{Re}(\lambda)>0$.

Proof To describe the relation (41), from (40) in (23), we see that

$$
\begin{aligned}
\mathcal{F}^{S}(w)= & \sqrt{\frac{2}{\pi}} \int_{0}^{\infty} \xi^{2 n+1} e^{-\mu \xi^{2}} \mathcal{Y}_{n}\left(\lambda \xi^{2} ; F, L\right) \sin (\xi w) d \xi \\
= & \sqrt{\frac{2}{\pi}} \sum_{r=0}^{n}(-n I)_{r}(F+(n-1) I)_{r} \frac{\left(-\lambda L^{-1}\right)^{r}}{r !} \\
& \times \int_{0}^{\infty} \xi^{2 n+2 r+1} e^{-\mu \xi^{2}} \sin (\xi w) d \xi \\
= & \sqrt{\frac{2}{\pi}} \sum_{r=0}^{n} \sum_{s=0}^{\infty}(-n I)_{r}(F+(n-1) I)_{r} \\
& \times \frac{(-1)^{s}(w)^{2 s+1}\left(-\lambda L^{-1}\right)^{r} \Gamma(n+r+s+3 / 2)}{2 \mu^{(n+r+s+3 / 2)}(2 s+1) ! r !} \\
= & \sqrt{\frac{1}{2 \pi} w \mu^{-(n+3 / 2)} \Gamma(n+3 / 2)} \\
& \times \sum_{r=0}^{n}(-n I)_{r}(F+(n-1) I)_{r}(n+3 / 2)_{r} \frac{\left(-\lambda(\mu L)^{-1}\right)^{r}}{r !} \\
& \times e^{\left(-w^{2} / 4 \mu\right) \sum_{s=0}^{n+r}\left\{\frac{(-n-r)_{s}}{s !(3 / 2)_{s}}\right\}\left(w^{2} / 4 \mu\right)^{s} .} \\
&
\end{aligned}
$$

After simplification, we obtain the desired result

$$
\begin{aligned}
\mathcal{F}^{S}(w)= & \frac{1}{2 \sqrt{2}} \mu^{-(2 n+3 / 2)}\left(\frac{-w^{2}}{4}\right)^{n} e^{\left(-w^{2} / 4 \mu\right)} \\
& \times \sum_{r=0}^{n}(-n I)_{r}(F+(n-1) I)_{r} \frac{\left(\lambda w\left(4 \mu^{2} L\right)^{-1}\right)^{r}}{r !} \\
& \left.\times{ }_{2} \mathcal{H}_{0}\left[\begin{array}{c}
-n-r,-\frac{1}{2}-n-r \\
-
\end{array}\right]-4 \mu / w^{2}\right] .
\end{aligned}
$$

This completes the proof of Theorem 3.6. 
Theorem 3.7 Let $S$ and $F$ be positive stable and commuting matrices in $\mathbb{C}^{n \times n}$. For the function

$$
f(\xi)=\xi^{S+\frac{1}{2} I} e^{-\mu \xi^{2}} \mathcal{Y}_{n}\left(1 ; F, \mu \xi^{2}\right)
$$

we have

$$
\begin{aligned}
\mathcal{F}^{c}(w)= & (-1)^{n} \sqrt{\frac{\pi}{2}} \mu^{-(S / 4+1 / 4)}(S / 2+F+3 / 4 I)_{n} \\
& \times \Gamma^{-1}((n-3 / 4) I-S / 2) \csc [\pi(S / 2+1 / 4 I)] \\
& \times{ }_{2} \mathcal{H}_{2}\left[\begin{array}{c}
(7 / 4-n) I+S / 2,(3 / 4-n) I+S / 2-F \\
\frac{1}{2}, 3 / 4 I+S / 2+F
\end{array}-w^{2} / 4 \mu\right],
\end{aligned}
$$

where $w, \mu \in \mathbb{C}$ are such that $\operatorname{Re}(w)>0, \operatorname{Re}(\mu)>0$, and $\widetilde{\alpha}(S+3 / 2 I)<2 n+\widetilde{\alpha}(2 F)$, as well as

$$
\begin{aligned}
\mathcal{F}^{S}(w)= & w(-1)^{n} \sqrt{\frac{\pi}{2}} \mu^{-(S / 2+3 / 4)}(S / 2+F-1 / 4 I)_{n} \\
& \times \Gamma^{-1}((n+1 / 4) I-S / 2) \csc [\pi(S / 2+3 / 4 I)] \\
& \times{ }_{2} \mathcal{H}_{2}\left[\begin{array}{c}
(3 / 4-n) I+S / 2,(n-1 / 4) I+S / 2+F \\
\frac{3}{2} I,-1 / 4 I+S / 2+F
\end{array} ; w^{2} / 4 \mu\right],
\end{aligned}
$$

where $w, \mu \in \mathbb{C}$ are such that $\operatorname{Re}(w)>0, \operatorname{Re}(\mu)>0$, and $\widetilde{\alpha}(S+1 / 2 I)>0, \widetilde{\alpha}(S)<2 n+1 / 2$.

Proof To demonstrate the truth of these results, making use of (22) with (42), we observe that

$$
\begin{aligned}
\mathcal{F}^{c}(w)= & \sqrt{\frac{2}{\pi}} \int_{0}^{\infty} \xi^{S+\frac{1}{2} I} e^{-\mu \xi^{2}} \mathcal{Y}_{n}\left(1 ; F, \mu \xi^{2}\right) \cos (\xi w) d \xi \\
= & \sqrt{\frac{1}{2 \pi}} \sum_{r=0}^{n} \sum_{s=0}^{\infty}(-n I)_{r}(F+(n-1) I)_{r} \frac{(-1 / \mu)^{r}\left(-w^{2}\right)^{s}}{r !(2 s) !} \\
& \times \int_{0}^{\infty} \xi^{S / 2+(1 / 4-r+s-1) I} e^{-\mu \xi} d \xi \\
= & \sqrt{\frac{1}{2 \pi}} \sum_{r=0}^{n} \sum_{s=0}^{\infty}(-n I)_{r}(F+(n-1) I)_{r} \frac{(-1 / \mu)^{r}\left(-w^{2}\right)^{s}}{r !(2 s) !} \\
& \times \mu^{(-S / 2+(r-s-1 / 4) I)} \Gamma(S / 2+(s-r+1 / 4) I) .
\end{aligned}
$$

Thus, after a simplification, we find that

$$
\begin{aligned}
\mathcal{F}^{c}(w)= & \sqrt{\frac{1}{2 \pi}} \mu^{-(S / 2+1 / 4 I)} \Gamma(S / 2+1 / 4 I) \\
& \times \sum_{s=0}^{\infty}(S / 2+1 / 4 I)_{s} \Gamma(-S / 2-F-(3 / 4+s-1) I) \Gamma(-S / 2-(3 / 4+s) I) \\
& \times \Gamma^{-1}(-S / 2-F-(3 / 4+s+n-1) I)
\end{aligned}
$$




$$
\begin{aligned}
& \times \Gamma^{-1}(-S / 2-(3 / 4+s-n) I) \frac{\left(-w^{2} / 4 \mu\right)^{s}}{s !\left(\frac{1}{2}\right)_{s}} \\
= & \sqrt{\frac{1}{2 \pi}} \mu^{-(S / 2+1 / 4 I)} \Gamma(S / 2+1 / 4 I) \Gamma(-S / 2-3 / 4 I) \Gamma(-S / 2-F+1 / 4 I) \\
& \times \Gamma^{-1}(-S / 2-F+(1 / 4-n) I) \Gamma^{-1}(-S / 2-(3 / 4-n) I) \\
& \times \sum_{s=0}^{\infty}(S / 2+1 / 4 I)_{s}(S / 2+(7 / 2-n) I)_{s}(S / 2+F+(\mu / 4-n) I)_{s} \\
& \times\left[(S / 2+F+3 / 4 I)_{s}\right]^{-1}\left[(S / 2+1 / 4 I)_{s}\right]^{-1} \frac{\left(-w^{2} / 4 \mu\right)^{s}}{s !\left(\frac{1}{2}\right)_{s}} .
\end{aligned}
$$

The above equation gives the proof of (43).

In a similar way and by using (23) with (42), we can get the result in (44).

Hence, the demonstration of Theorem 3.7 is finished.

Theorem 3.8 Let $S, F$, and $L$ be commuting matrices in $\mathbb{C}^{n \times n}$. If

$$
f(\xi)=\xi^{S} \frac{1}{\left(\mu^{2}+\xi^{2}\right)} J_{S}(\lambda \xi) \mathcal{Y}_{n}\left(\xi^{2} ; F, L\right)
$$

then, we have

$$
\mathcal{F}^{S}(w)=\sqrt{\frac{2}{\pi}} \mu^{S-I} \sinh (\mu w) K_{S}(\lambda \mu) \mathcal{Y}_{n}\left(\mu^{2} ; F,-L\right),
$$

where $w, \mu, \lambda \in \mathbb{C}$ are such that $\operatorname{Re}(w)>0, \operatorname{Re}(\mu)>0, \operatorname{Re}(\lambda)>0$, and $S$ is a positive stable matrix in $\mathbb{C}^{n \times n}$ such that $-1<\tilde{\alpha}(S)<0, J_{S}(x)$ is the Bessel matrix function defined in (17) and $K_{S}(x)$ is the modified Bessel matrix function defined in (19).

Proof The proof of this result indeed follows from applying (23) on (45), we have

$$
\begin{aligned}
\mathcal{F}^{S}(w)= & \sqrt{\frac{2}{\pi}} \int_{0}^{\infty} \xi^{S} \frac{1}{\left(\mu^{2}+\xi^{2}\right)} J_{S}(\lambda \xi) \mathcal{Y}_{n}\left(\xi^{2} ; F, L\right) \sin (\xi w) d \xi \\
= & \sqrt{\frac{2}{\pi}} \sum_{r=0}^{n}(-n I)_{r}(F+(n-1) I)_{r} \frac{\left(-L^{-1}\right)^{r}}{r !} \\
& \times \int_{0}^{\infty} \xi^{(2 r+1) I-S} \frac{1}{\left(\mu^{2}+\xi^{2}\right)} J_{S}(\lambda \xi) \sin (\xi w) d \xi .
\end{aligned}
$$

Using the Fourier sine transform (see [18, p. 426]), we obtain

$$
\begin{aligned}
\mathcal{F}^{S}(w)= & \sqrt{\frac{2}{\pi}} \sum_{r=0}^{n}(-n I)_{r}(F+(n-1) I)_{r} \\
& \times \frac{L^{-r}}{r !} \mu^{(S+(2 r-1) I)} \sinh (\mu w) K_{S}(\mu \lambda) \\
= & \sqrt{\frac{2}{\pi}} \mu^{S-I} \sinh (\mu w) K_{S}(\mu \lambda) \sum_{r=0}^{n}(-n I)_{r}(F+(n-1) I)_{r} \frac{\left(\mu^{2} L^{-1}\right)^{r}}{r !} .
\end{aligned}
$$

This completes the proof of equation (46) asserted in Theorem 3.8. 
Similarly, we can arrive at the following result.

Theorem 3.9 Let $\mathcal{Y}_{n}\left(\xi^{2} ; F, L\right)$ be given in (15). For the function

$$
f(\xi)=\xi^{I-S} \frac{1}{\left(\mu^{2}+\xi^{2}\right)} J_{S}(\lambda \xi) \mathcal{Y}_{n}\left(\xi^{2} ; F, L\right),
$$

we have

$$
\mathcal{F}^{S}(w)=\sqrt{\frac{\pi}{2}} \mu^{-S} e^{(-\mu w)} J_{S}(\lambda w) \mathcal{Y}_{n}\left(-\mu^{2} ; F,-L\right),
$$

where $w, \mu, \lambda \in \mathbb{C}$ are such that $\operatorname{Re}(w)>0, \operatorname{Re}(\mu)>0, \operatorname{Re}(\lambda)>0, \operatorname{Re}(w)>\operatorname{Re}(\lambda)$, S is a positive stable matrix in $\mathbb{C}^{n \times n}$ such that $\widetilde{\alpha}(I-S)>0$, and $S, F$, and L are commuting matrices in $\mathbb{C}^{n \times n}$.

Theorem 3.10 Let $\mathcal{Y}_{n}\left(\xi^{2} ; F, L\right)$ be given in (15). For the function

$$
f(\xi)=\left(\lambda^{2}+\xi^{2}\right)^{-\left(S+\frac{1}{2} I\right)} \mathcal{Y}_{n}\left(\xi^{2} ; F, L\right)
$$

we have

$$
\begin{aligned}
\mathcal{F}^{c}(w)= & \frac{\sqrt{8}}{\pi}(2 F)^{-S} \Gamma^{-1}\left(S+\frac{1}{2} I\right) \\
& \times \sum_{r=0}^{n}(-n I)_{r}(F+(n-1) I)_{r} \frac{L^{-r}}{r !} \frac{d^{2 r}\left(w^{S} K_{S}(\lambda w)\right)}{d w^{2 r}},
\end{aligned}
$$

where $w, \lambda \in \mathbb{C}$ are such that $\operatorname{Re}(w)>0, \operatorname{Re}(\lambda)>0, S$ is a positive stable matrix in $\mathbb{C}^{n \times n}$ such that $\tilde{\alpha}(S)>-\frac{1}{2}, K_{S}(x)$ is modified Bessel matrix function defined in (19), and $S, F$, and $L$ are commuting matrices in $\mathbb{C}^{n \times n}$.

Proof In order to establish the result (50), with the help of the Lemma 2.3, we get

$$
\begin{aligned}
\mathcal{F}^{c}(w)= & \sqrt{\frac{2}{\pi}} \int_{0}^{\infty}\left(\lambda^{2}+\xi^{2}\right)^{-\left(S+\frac{1}{2} I\right)} \mathcal{Y}_{n}\left(\xi^{2} ; F, L\right) \cos (w \xi) d \xi \\
= & \sqrt{\frac{2}{\pi}} \sum_{r=0}^{n}(-n I)_{r}(F+(n-1) I)_{r} \frac{(-L)^{-r}}{r !} \\
& \times \int_{0}^{\infty} \xi^{2 r}\left(\lambda^{2}+\xi^{2}\right)^{-\left(S+\frac{1}{2} I\right)} \cos (w \xi) d \xi \\
= & \sqrt{\frac{2}{\pi}} \sum_{r=0}^{n}(-n I)_{r}(F+(n-1) I)_{r} \frac{(-L)^{-r}}{r !} \\
& \times \sqrt{\frac{2}{\pi}}(-1)^{r} \sqrt{2} \Gamma^{-1}\left(S+\frac{1}{2} I\right)(2 F)^{-S} \frac{d^{2 r}\left(w^{S} \mathbf{K}_{S}(\lambda w)\right)}{d w^{2 r}} .
\end{aligned}
$$

This completes the proof.

Similarly, we can arrive at the following result. 
Theorem 3.11 Let $\mathcal{Y}_{n}\left(\xi^{2} ; F, L\right)$ be given in (15). For the function

$$
f(\xi)=\xi\left(\lambda^{2}+\xi^{2}\right)^{-\left(n+\frac{1}{2}\right)} \mathcal{Y}_{n}\left(\xi^{2} ; F, L\right)
$$

we have

$$
\mathcal{F}^{c}(w)=\frac{-\sqrt{8}}{\pi \Gamma\left(n+\frac{1}{2}\right)}(2 F)^{-n} \frac{d^{2 r+1}\left(w^{n} K_{n}(\lambda w)\right)}{d w^{2 r+1}},
$$

where $w, \lambda \in \mathbb{C}$ are such that $\operatorname{Re}(w)>0, \operatorname{Re}(\lambda)>0$, and $K_{n}(w)$ is the modified Bessel function defined in [18].

Theorem 3.12 Let $\mathcal{Y}_{n}(\xi ; F, L)$ be given in (15). For the function

$$
f(\xi)=\xi^{-1} e^{-\lambda / \xi} \mathcal{Y}_{n}(\xi ; F, L)
$$

we have

$$
\begin{aligned}
\mathcal{F}^{c}(w)= & \sqrt{\frac{2}{\pi}} \sum_{r=0}^{n}(-n I)_{r}(F+(n-1) I)_{r} \frac{(-L)^{-r}(w / \lambda)^{\frac{r}{2}}}{r !} \\
& \times\left\{e^{i \frac{\pi r}{4}} K_{r}(2 \sqrt{i \lambda w})+e^{-i \frac{\pi r}{4}} K_{r}(2 \sqrt{-i \lambda w})\right\}
\end{aligned}
$$

where $w, \lambda \in \mathbb{C}$ are such that $\operatorname{Re}(w)>0$ and $\operatorname{Re}(\lambda)>0, \xi \in \mathbb{C} \backslash\{0\}$, and $K_{r}(w)$ is the modified Bessel function defined in [18].

Proof Using (15) and (53) in (22), we observe that

$$
\begin{aligned}
\mathcal{F}^{c}(w)= & \sqrt{\frac{2}{\pi}} \int_{0}^{\infty} \xi^{-1} e^{-\lambda / \xi} \mathcal{Y}_{n}(\xi ; F, L) \cos (w \xi) d \xi \\
= & \sqrt{\frac{2}{\pi}} \sum_{r=0}^{n}(-n I)_{r}(F+(n-1) I)_{r} \frac{(-L)^{-r}}{r !} \\
& \times \int_{0}^{\infty} \xi^{r-1} e^{-\lambda / \xi} \cos (w \xi) d \xi .
\end{aligned}
$$

Applying the formula in [18, p. 403], we see that

$$
\begin{aligned}
\mathcal{F}^{c}(w)= & \sqrt{\frac{2}{\pi}} \sum_{r=0}^{n}(-n I)_{r}(F+(n-1) I)_{r} \frac{(-L)^{-r}(w / \lambda)^{\frac{r}{2}}}{r !} \\
& \times\left\{e^{i \frac{\pi r}{4}} K_{r}(2 \sqrt{i \lambda w})+e^{-i \frac{\pi r}{4}} K_{r}(2 \sqrt{-i \lambda w})\right\}
\end{aligned}
$$

This completes the proof of Theorem 3.12.

\section{Conclusion}

In this manuscript, motivated by the recent studies and developments of the integral transforms with various special matrix functions, including the matrix orthogonal polynomials as kernels and their applications $[14,17,49,50]$, we introduce some Fourier cosine and 
sine transforms of generalized Bessel matrix polynomials, together with certain elementary matrix functions, as well as exponential and Bessel functions. It is obvious that the results presented here, which involve certain matrices in $\mathbb{C}^{n \times n}$, reduce to the corresponding scalar ones when $n=1$.

In fact, a remarkably large number of Fourier cosine and sine formulas involving a variety of functions have been published (see, e.g., [51, pp. 7-108]). In this connection, we conclude this manuscript by posing the following problem for further investigation.

Open problem. Try to give matrix versions of the results for Fourier integral transforms (or formulas) involving a variety of special functions (see, e.g., [51, pp. 7-108]).

\section{Acknowledgements}

The first author extends his appreciation to the Deanship of Scientific Research at King Khalid University for funding this work through research groups program under grant (R.G.P.1/15/42).

The second-named author would like to thank the Academy of Scientific Research and Technology (ASRT), Egypt for supporting him through Grant (6407).

\section{Funding}

Deanship of Scientific Research at King Khalid University.

\section{Availability of data and materials}

The data that support the findings of this study are available from the authors, upon request.

\section{Competing interests}

The authors declare that they have no competing interests.

Authors' contributions

All authors jointly worked on the results and they read and approved the final manuscript.

\section{Author details}

'Department of Mathematics, College of Science, King Khalid University, P.O. Box 9004, 61413, Abha, Saudi Arabia. ${ }^{2}$ Department of Mathematics, Faculty of Science, South Valley University, 83523 Qena, Egypt. ${ }^{3}$ The Academy of Scientific Research and Technology (ASRT), Cairo, 82524, Egypt.

\section{Publisher's Note}

Springer Nature remains neutral with regard to jurisdictional claims in published maps and institutional affiliations.

Received: 30 January 2021 Accepted: 31 August 2021 Published online: 15 September 2021

\section{References}

1. Ismaila, M., Koelink, E., Román, P.: Matrix valued Hermite polynomials, Burchnall formulas and non-Abelian Toda lattice. Adv. Appl. Math. 110, 235-269 (2019)

2. Dwivedi, R., Sahai, V.: Lie algebras of matrix difference differential operators and special matrix functions. Adv. Appl. Math. 122, 102-109 (2021)

3. Iserles, A., Webb, M.: A family of orthogonal rational functions and other orthogonal systems with a skew-Hermitian differentiation matrix. J. Fourier Anal. Appl. 26, 19 (2020)

4. Abdalla, M.: Special matrix functions: characteristics, achievements and future directions. Linear Multilinear Algebra 68, 1-28 (2020)

5. Abdalla, M., Boulaaras, S.: Analytical properties of the generalized heat matrix polynomials associated with fractional calculus. J. Funct. Spaces 2021, Article ID 4065606 (2021)

6. Rodman, L.: Orthogonal matrix polynomials. In: Nevai, P. (ed.) Orthogonal Polynomials Theory and Practice. NATOASI Series, vol. 295. Kluwer Academic, Dordrecht (1990)

7. Zayed, M., Abul-Ez, M., Abdalla, M., Saad, N.: On the fractional order Rodrigues formula for the shifted Legendre-type matrix polynomials. Mathematics 8, 136 (2020)

8. Kim, I.-S.: Semilinear problems involving nonlinear operators of monotone type. Results Nonlinear Anal. 2, 25-35 (2019)

9. Abdalla, M., Hidan, M.: Analytical properties of the two-variables Jacobi matrix polynomials with applications. Demonstr. Math. 54, 178-188 (2021)

10. Izadi, M., Cattani, C.: Generalized Bessel polynomial for multi-order fractional differential equations. Symmetry 12 , 1260 (2020)

11. Tcheutia, D.: Nonnegative linearization coefficients of the generalized Bessel polynomials. Ramanujan J. 48, 217-231 (2019)

12. Abdalla, M., Abul-Ez, M., Morais, J.: On the construction of generalized monogenic Bessel polynomials. Math. Methods Appl. Sci. 40, 1-14 (2018)

13. Abul-Dahab, M., Abul-Ez, M., Kishka, Z., Constales, D.: Reverse generalized Bessel matrix differential equation, polynomial solutions, and their properties. Math. Methods Appl. Sci. 38, 1005-1013 (2015) 
14. Abdalla, M., Akel, M., Choi, J.: Certain matrix Riemann-Liouville fractional integrals associated with functions involving generalized Bessel matrix polynomials. Symmetry 13, 622 (2021)

15. Abdalla, M., Hidan, M.: Fractional orders of the generalized Bessel matrix polynomials. Eur. J. Pure Appl. Math. 10, 995-1004 (2017)

16. Abdalla, M.: Operational formula for the generalized Bessel matrix polynomials. J. Mod. Methods Numer. Math. 8, 156-163 (2017)

17. Hidan, M., Akel, M., Boulaaras, S., Abdalla, M.: On behavior Laplace integral operators with generalized Bessel matrix polynomials and related functions. J. Funct. Spaces 2021, Article ID 9967855 (2021)

18. Magnus, W., Oberhettinger, F., Soni, R.: Formulas and Theorems for the Special Functions of Mathematical Physics. Springer, Berlin (1966)

19. Luchko, Y.: Some schemata for applications of the integral transforms of mathematical physics. Mathematics 7, 254 (2019)

20. Debnath, L., Bhatta, D.: Integral Transforms and Their Applications, 3rd edn. Chapman \& Hall, London (2015)

21. Lazreg, J.E., Abbas, S., Benchohra, M., Karapinar, E.: Impulsive Caputo-Fabrizio fractional differential equations in b-metric spaces. Open Math. 19, 363-372 (2021). https://doi.org/10.1515/math-2021-0040

22. Adiguzel, R.S., Aksoy, U., Karapinar, E., Erhan, I.M.: On the solutions of fractional differential equations via Geraghty type hybrid contractions. Appl. Comput. Math. 20, 313-333 (2021)

23. Adiguzel, R.S., Aksoy, U., Karapinar, E., Erhan, I.M.: Uniqueness of solution for higher-order nonlinear fractional differential equations with multi-point and integral boundary conditions. Rev. R. Acad. Cienc. Exactas Fís. Nat., Ser. A Mat. 115, 155 (2021). https://doi.org/10.1007/s13398-021-01095-3

24. Adiguzel, R.S., Aksoy, U., Karapinar, E., Erhan, I.M.: On the solution of a boundary value problem associated with a fractional differential equation. Math. Methods Appl. Sci. (2020). https://doi.org/10.1002/mma.665

25. Ameen, R., Köse, H., Jarad, F.: On the discrete Laplace transform. Results Nonlinear Anal. 2(2), 61-70 (2019)

26. Chakraborty, K., Kanemitsu, S., Tsukada, H.: Applications of the Beta-transform. Šiauliai Math. Semin. 10, 5-28 (2015)

27. Jarada, F., Abdeljawad, T.: A modified Laplace transform for certain generalized fractional operators. Results Nonlinear Anal. 1, 88-98 (2018)

28. Redhwan, S.S., Shaikh, S.L., Abdo, M.S.: Some properties of Sadik transform and its applications of fractional-order dynamical systems in control theory. Adv. Theory Nonlinear Anal. Appl. 4, 51-66 (2020)

29. Sunthrayuth, P., Zidan, A.M., Yao, S., Shah, R., Inc, M.: The comparative study for solving fractional-order Fornberg-Whitham equation via p-Laplace transform. Symmetry 13, 784 (2021). https://doi.org/10.3390/sym13050784

30. Nonlaopo, K., Alsharif, A.M., Zidan, A.M., Khan, A., Hamed, Y.S., Shah, R.: Numerical investigation of fractional-order Swift-Hohenberg equations via a Novel transform. Symmetry 13, 1263 (2021). https://doi.org/10.3390/sym13071263

31. Folland, G.: Fourier Analysis and Its Applications. Wadsworth, Pacific Grove (1992)

32. Serov, V.: Fourier Series, Fourier Transform and Their Applications to Mathematical Physics. Springer, Berlin (2017)

33. Nicola, F., Trapasso, S.: A note on the HRT conjecture and a new uncertainty principle for the short-time Fourier transform. J. Fourier Anal. Appl. 26, 68 (2020)

34. Urieles, A., Ramrez, W., Ortega, M., Bedoya, D.: Fourier expansion and integral representation generalized Apostol-type Frobenius-Euler polynomials. Adv. Differ. Equ. 2020, 534 (2020)

35. Ghodadra, B., Fülöp, V.: On the order of magnitude of Walsh-Fourier transform. Math. Bohem. 145, $265-280$ (2020)

36. Bergold, P., Lasser, C.: Fourier series windowed by a bump function. J. Fourier Anal. Appl. 26, 65 (2020)

37. Al-Lail, M., Qadir, A.: Fourier transform representation of the generalized hypergeometric functions with applications to the confluent and Gauss hypergeometric functions. Appl. Math. Comput. 263, 392-397 (2015)

38. Osihnker, B.: On matrix Fourier expansions. Proc. MICHM, Moscow 64, 79-92 (1975)

39. Osihnker, B.: Fourier series in orthonormal matrix polynomials. Izvestija VUZ. Mathematika 32, 71-83 (1988)

40. Jóbdar, L., Navarro, E., Defez, E.: On the best approximation matrix problem and matrix Fourier series. Approx. Theory Appl. 13, 88-98 (1997)

41. Defez, E., Jóbdar, L.: On the best approximation matrix problem for integrable matrix functions. Approx. Theory Appl. $16,56-71(2000)$

42. Groenevelt, W., Koelink, E.: A hypergeometric function transform and matrix-valued orthogonal polynomials. Constr. Approx. 38, 277-309 (2013)

43. Yildiz, Ş:: On application of matrix summability to Fourier series. Math. Methods Appl. Sci. 41, 664-670 (2018)

44. Horn, R., Johnson, C.: Topics in Matrix Analysis. Cambridge University Press, Cambridge (1991)

45. Khammash, G., Agarwal, P., Choi, J.: Extended k-gamma and k-beta functions of matrix arguments. Mathematics 8 , 1715 (2020)

46. Abdalla, M.: Further results on the generalised hypergeometric matrix functions. Int. J. Comput. Sci. Math. 10, 1-10 (2019)

47. Jódar, L., Company, R., Navarro, E.: Bessel matrix functions: explicit solution of coupled Bessel type equations. Util. Math. 46, 129-141 (1994)

48. Sastre, J., Jódar, L.: Asymptotics of the modified Bessel and incomplete gamma matrix functions. Appl. Math. Lett. 16, 815-820 (2003)

49. Abdalla, M., Boulaaras, S., Akel, M.: On Fourier-Bessel matrix transforms and applications. Math. Methods Appl. Sci. (2021). https://doi.org/10.1002/mma.7489

50. Abdalla, M.: On Hankel transforms of generalized Bessel matrix polynomials. AlMS Math. 6, 6122-6139 (2021)

51. Erdélyi, A., Magnus, W., Oberhettinger, F., Tricomi, F.G.: Tables of Integral Transforms, Vol. I. McGraw-Hill, New York (1954) 